\title{
The 2010 outbreak of poliomyelitis in Tajikistan: epidemiology and lessons learnt
}

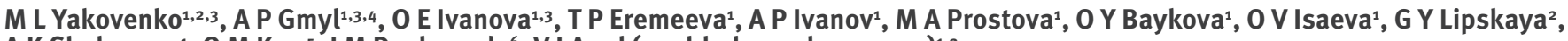

A K Shakaryan ${ }^{1}, \mathrm{O}_{\mathrm{M} \mathrm{Kew}}^{5}$, J M Deshpande ${ }^{6}$, V I Agol (agol.belozersky.msu.ru) , $^{1,2}$

1. M.P. Chumakov Institute of Poliomyelitis and Viral Encephalitides, Russian Academy of Medical Sciences, Moscow, Russia

2. A.N. Belozersky Institute of Physical-Chemical Biology, M.V. Lomonosov Moscow State University, Moscow, Russia

3. These authors contributed equally to this study

4. Faculty of Biology, M.V. Lomonosov Moscow State University, Moscow, Russia

5. Centers for Disease Control and Prevention, Atlanta, Georgia, United States

6. Enterovirus Research Centre, Indian Council of Medical Research, Mumbai, India

Citation style for this article:

Yakovenko ML, Gmyl AP, Ivanova OE, Eremeeva TP, Ivanov AP, Prostova MA, Baykova OY, Isaeva OV, Lipskaya GY, Shakaryan AK, Kew OM, Deshpande JM, Agol VI. The 2010 outbreak of poliomyelitis in Tajikistan: epidemiology and lessons learnt. Euro Surveill. 2014;19(7): pii=20706. Available online: http://www. eurosurveillance.org/ViewArticle.aspx?Articleld =20706

A large outbreak of poliomyelitis, with 463 laboratoryconfirmed and 47 polio-compatible cases, took place in 2010 in Tajikistan. Phylogenetic analysis of the viral VP1 gene suggested a single importation of wild poliovirus type 1 from India in late 2009, its further circulation in Tajikistan and expansion into neighbouring countries, namely Kazakhstan, Russia, Turkmenistan and Uzbekistan. Whole-genome sequencing of 14 isolates revealed recombination events with enterovirus $\mathrm{C}$ with cross-overs within the $\mathrm{P}_{2}$ region. Viruses with one class of recombinant genomes co-circulated with the parental virus, and representatives of both caused paralytic poliomyelitis. Serological analysis of 327 sera from acute flaccid paralysis cases as well as from patients with other diagnoses and from healthy people demonstrated inadequate immunity against polio in the years preceding the outbreak. Evidence was obtained suggesting that vaccination against poliomyelitis, in rare cases, may not prevent the disease. Factors contributing to the peculiarities of this outbreak are discussed. The outbreak emphasises the necessity of continued vaccination against polio and the need, at least in risk areas, of quality control of this vaccination through well planned serological surveillance.

\section{Introduction}

A tremendous decrease in the incidence of paralytic poliomyelitis has occurred since 1988 , when the World Health Organization (WHO) launched the Global Polio Eradication Initiative [1]. Nevertheless, the originally set goal to eradicate polio worldwide by the year 2000 was not achieved. Two major problems impede the accomplishment of this task, (i) continuous circulation of wild polioviruses (WPV) in a few countries and importation of these viruses to other countries [2], and (ii) the ability of Sabin vaccine viruses to circulate for a long time, with the propensity to acquire phenotypical properties, including neurovirulence and transmissibility, similar to those of their wild-type counterparts [3].
An outbreak of poliomyelitis occurred in Tajikistan in 2010 [4]. An increase in the incidence of acute flaccid paralysis (AFP) was registered, starting early in that year. The number of AFP cases increased modestly in February and March, and peaked between mid-April and mid-May (Figure 1A). Twenty-nine fatal cases were registered. The outbreak was caused by WPV1 originating from India. To curb the outbreak, four rounds of supplementary immunisation activities with monovalent type 1 oral poliovirus vaccine (mOPV) were carried out in May (4-8 and 18-22 May) and June (1-5 and 15-19 June), followed by two rounds of immunisation with trivalent OPV (tOPV) on 4-8 October and 8-12 November 2010. In addition, mop-up vaccination with mOPV was carried out in 34 districts on 13-17 September 2010 [5]. As a result, the AFP incidence declined, and the last WPV 1 was isolated from an AFP patient on 4 July 2010. The total number of reported AFP cases in 2010 was 715 . The most affected cohort ( $43.8 \%)$ were one to five year-old children. The majority of the cases were registered in the most populated south-western districts of Tajikistan (Figure 2A). From Tajikistan, the outbreak spread to neighbouring countries in the same year (Kazkhstan, Russia, Turkmenistan and Uzbekistan). This polio epidemic was one of the largest among those recently caused by importation of WPV.

Here we present a molecular-epidemiological analysis of this outbreak. The nature and the origin of the virus were ascertained and its evolution was characterised. We have established the conditions favouring the outbreak and discuss implications for the worldwide efforts to combat poliomyelitis.

\section{Methods}

\section{Virological investigations}

Virus isolation from stools and typing were performed by standard methods [6]. Intratypic differentiation (ITD) was carried out by ELISA with cross-absorbed 


\section{FIGURE 1}

Cases of acute flaccid paralysis and laboratory-confirmed poliomyelitis, as deduced from our virological analyses and epidemiological information accompanying faecal samples, Tajikistan, $2010(\mathrm{n}=715)$

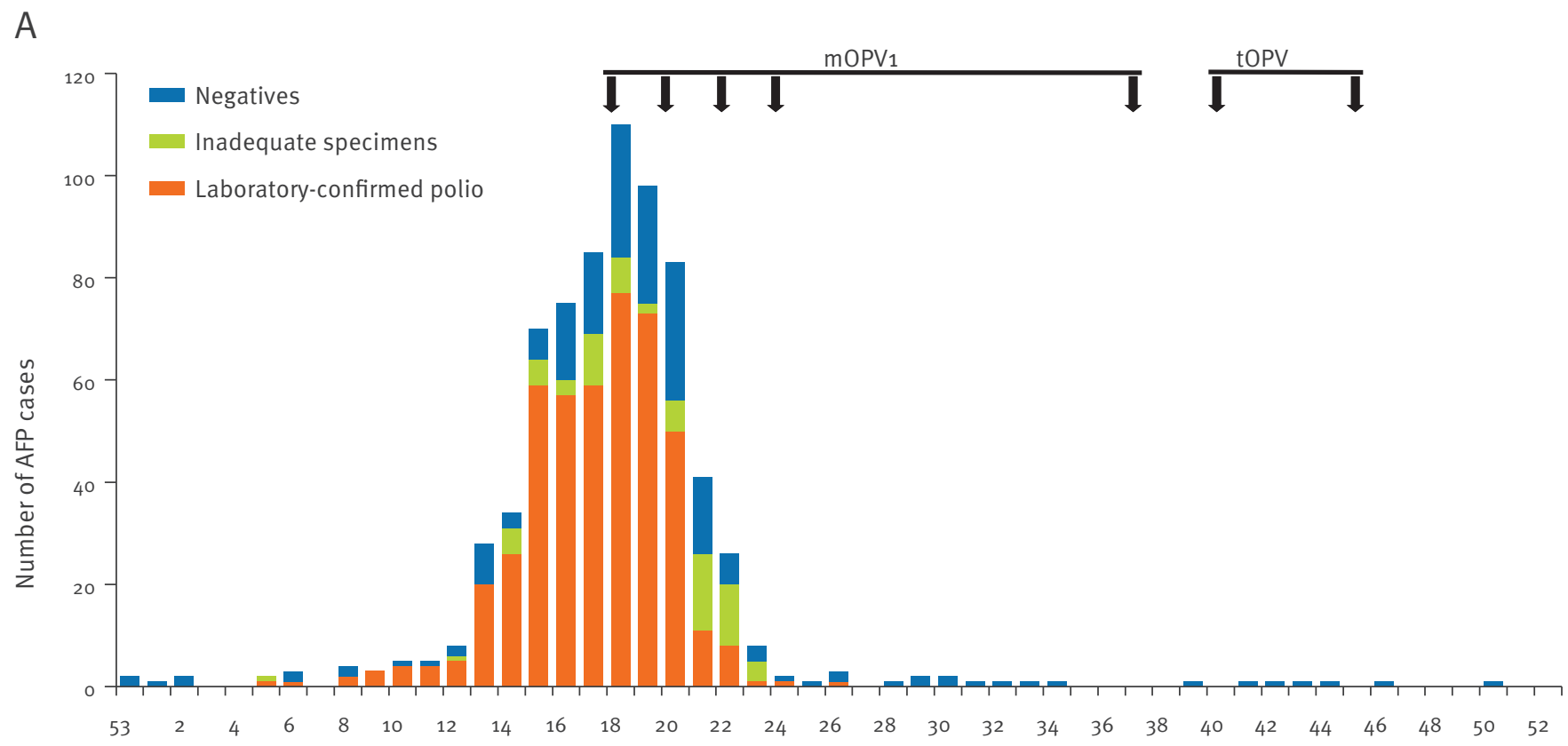

Week 2010

B

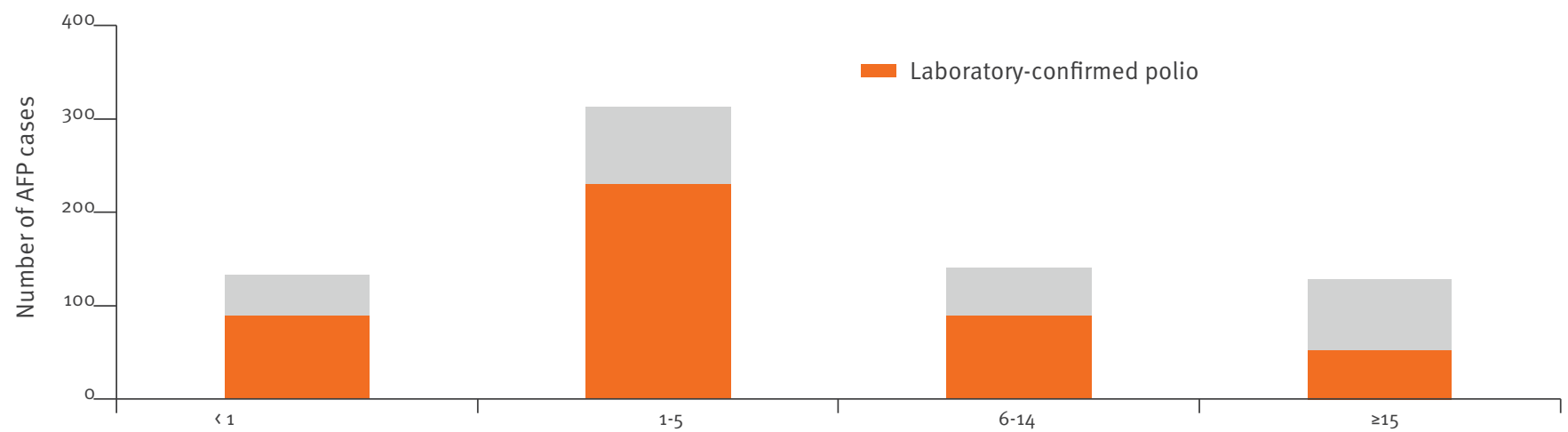

Age in years

AFP: acute flaccid paralysis; mOPV: monovalent type 1 Sabin vaccine; tOPV: trivalent Sabin vaccine.

(A) The time course of acute flaccid paralysis incidence and laboratory-confirmed poliomyelitis. Arrows indicate timing of mass immunisation with monovalent type 1 and trivalent Sabin vaccines [5]. The target groups during the first two rounds were children up to the age of six years, and in the subsequent rounds children under the age of 15 years.

(B) Age distribution of acute flaccid paralysis and confirmed poliomyelitis cases. 


\section{FIGURE 2}

Geographical distribution of acute flaccid paralysis and poliomyelitis cases in Tajikistan $(\mathrm{n}=715)$ and neighbouring countries $(\mathrm{n}=25)$ in 2010

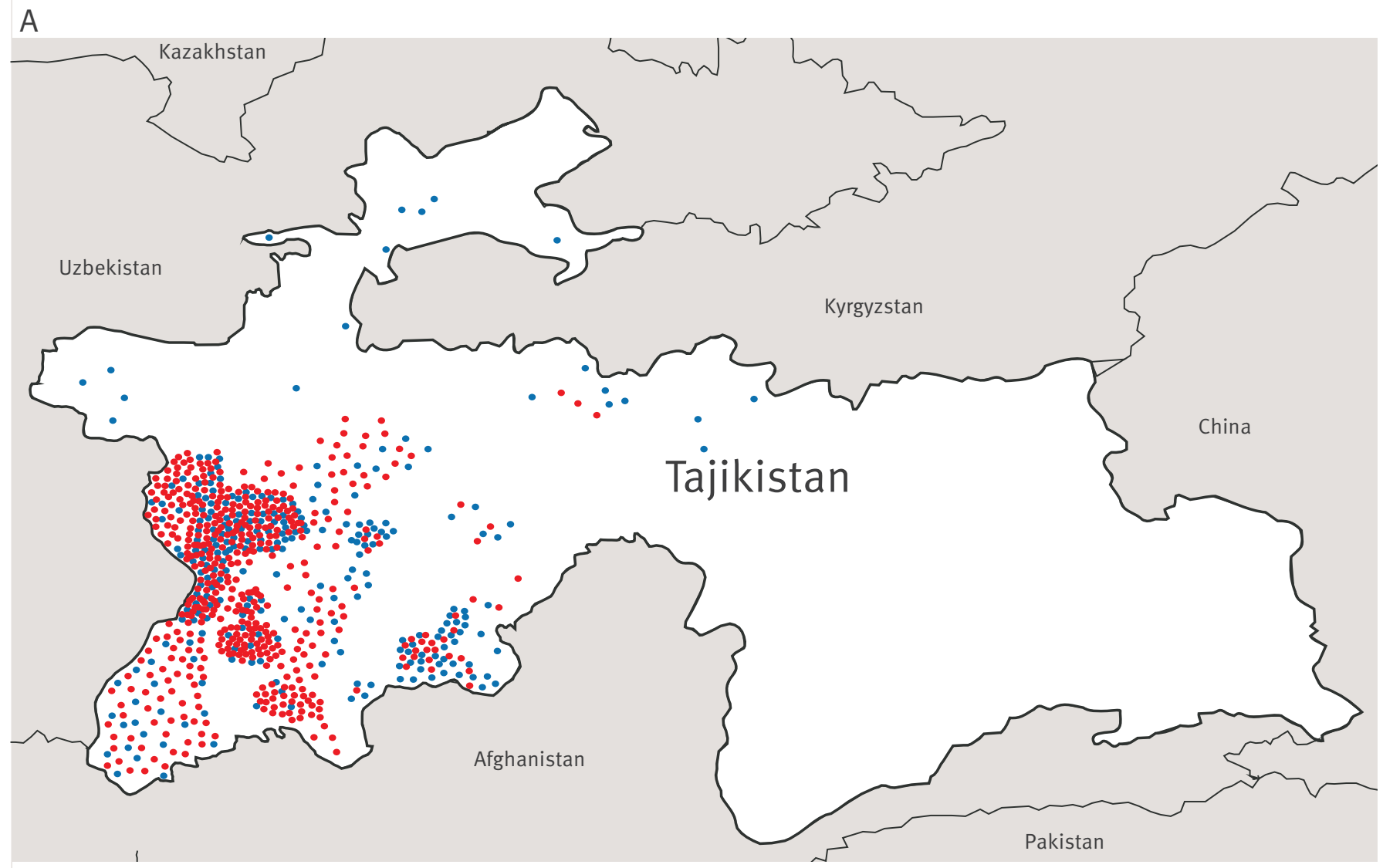

B

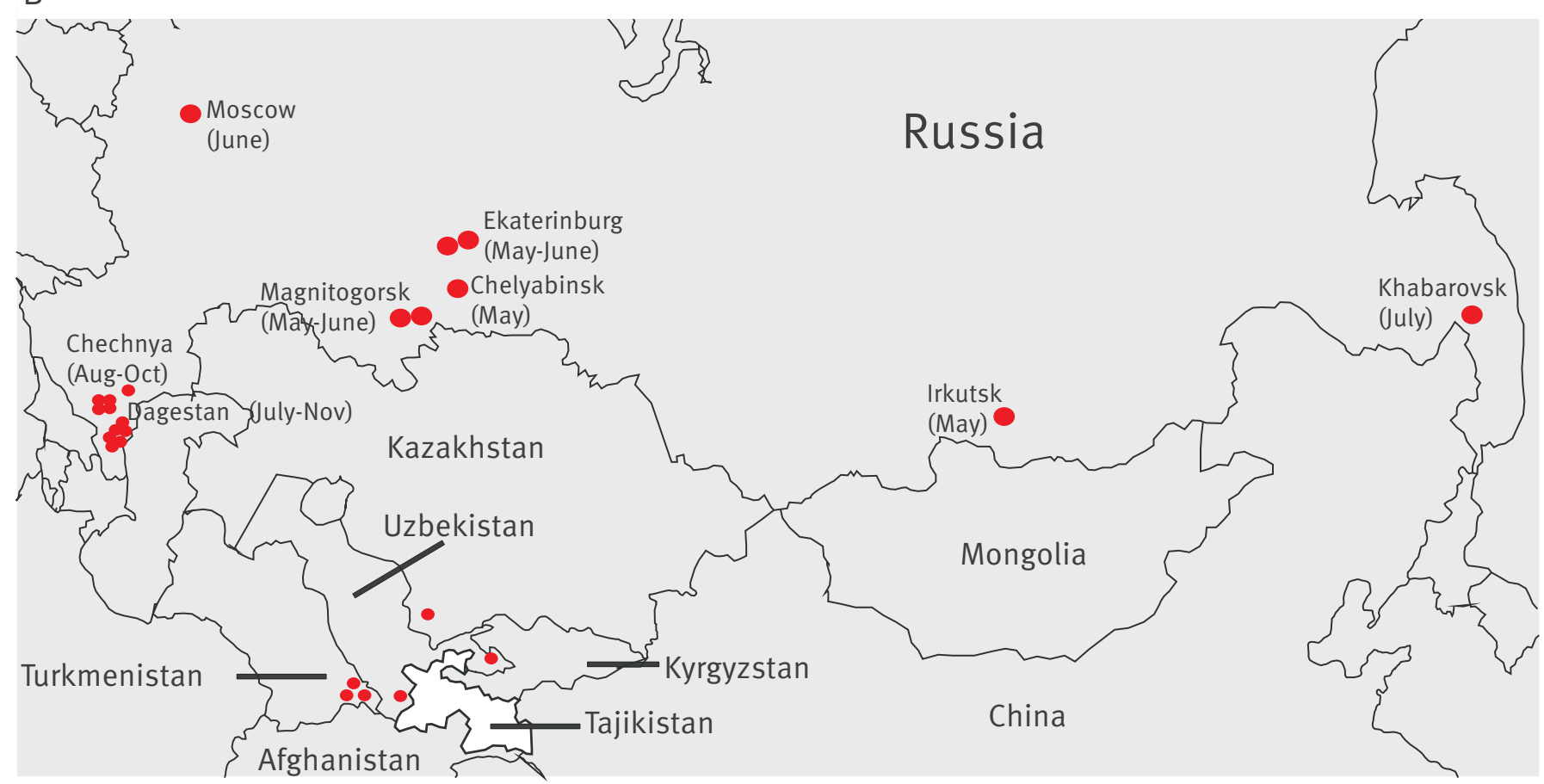

(A) Laboratory-confirmed cases due to wild poliovirus type 1 and polio-compatible (red dots) and other acute flaccid paralysis (blue dots) cases in Tajikistan. The dots are placed randomly within each district (borders not shown).

(B) Polio cases in Russia and other neighbouring countries due to wild poliovirus type 1 importation (red dots, with unspecific location within Kazakhstan, Turkmenistan and Uzbekistan or within each federal subject in the case of Russia).

The territory of Tajikistan is marked in white and represents the epicentre of the outbreak. 
polyclonal antisera (kindly provided by Dr HGAM van der Avoort, National Institute of Public Health and Environmental Protection (RIVM), the Netherlands) $[6,7]$ and real-time RT-PCR [8]. For sequencing, poliovirus RNA was extracted by phenol-chloroform from infected cells and was reverse-transcribed using random hexamer primers (Syntol) and SuperScriptll reverse transcriptase (Invitrogen). The CDNA of the region encoding the capsid protein VP1 was amplified by PCR using primers $Y 7 R$ and Q8 [9]. For full-genome sequencing, cDNA of eight overlapping genomic fragments were amplified by PCR (the primer sequences are available upon request). The products were purified with QiaQuick DNA Purification System (Qiagen). Sequencing was performed by using a Beckman Coulter Seq 8000 or ABI 3130 Genetic Analyzer.

\section{Serological assays}

Poliovirus-neutralising antibodies in human sera were determined by microneutralisation [10]. Sera with neutralising antibody titres $<1: 8$ were considered negative. Sabin 1-specific IgM and IgG were detected by ELISA [11].

\section{Origin of samples}

Stool samples from AFP cases were collected in Kazakhstan, Russia, Tajikistan, Turkmenistan, and Uzbekistan in accordance with the WHO Guidelines [10]. Stool samples from healthy close contacts of cases in Russia were collected following the national recommendations for investigation of suspected polio cases. The stool and sera specimens were collected [10] by local public health workers. Personal information (clinical data, vaccination status, age, location, etc) were retrieved from the documents accompanying the samples.

\section{Bioinformatics methods}

Multiple alignments, the estimation of the degree of synonymous nucleotide divergence and of the similarity of deduced amino acid sequences with the WPV sequences available in the GenBank were performed as described [12]. Bayesian phylogenetic analysis was conducted using the software BEAST 1.7.4 [13]. The general time-reversible substitution model with four gamma categories and invariant sites was used for calculations. Codons were grouped into three partitions and the substitution model was unlinked across codon positions. Markov chains were run for $2 \times 10^{8}$ generations, sampled every 5,000 generations, and executed three times to ensure adequate mixing and stationarity. The first 4,000 samples were discarded. The effective sample size, inspected with Tracer v1.5 (http:// tree.bio.ed.ac.uk/software/tracer/), of all parameters was larger than 200 , indicating that stationarity was achieved. An analysis of the marginal likelihoods indicated that the constant molecular clock and expansion population growth model $\left(\log ^{10} \mathrm{BF} / 2\right)$ was the best for our data set. For the SimPlot analysis, default settings were used [14].
Nucleotide sequence accession numbers

The nucleotide sequences determined in this study are available from GenBank, accession numbers KC800662-KC800683, KC812248-KC812257 and KC880365-KC880521.

\section{Results}

The causative agent of the outbreak, its origin and evolution

Faecal samples $(n=1,003)$ from 644 AFP cases were investigated, and WPV 1 was isolated from 463 patients (Figure $1 \mathrm{~A}$ ), who were therefore diagnosed as poliomyelitis cases. Of those 463, 29 cases were fatal. The age distribution of poliomyelitis cases paralleled that of AFP cases (Figure $1 \mathrm{~B}$ ). It is noteworthy that a significant proportion of poliomyelitis victims (including three fatal cases) were 15 years-old or older.

Specimens (two faecal samples each) from 181 AFP cases were poliovirus-negative. Specimens from an additional 69 AFP cases were unavailable and two specimens were delivered in inadequate condition. Forty-seven of these cases were eventually classified as 'polio-compatible' by the National Expert Committee in Tajikistan.

Non-polio enteroviruses were isolated from seven AFP cases, including five polio-negative cases (Echovirus 7, Coxsackievirus $B 1$ and three non-typable human enteroviruses) and two victims of poliomyelitis (Echovirus 13 and one non-typable, both in mixtures with WPV1).

We sequenced the capsid protein $\mathrm{VP}_{1}$ coding regions of 116 WPV $_{1}$ isolated from poliomyelitis cases from February to July 2010 in Tajikistan as well as the 37 isolates obtained from 25 poliomyelitis cases and eight healthy persons in April to November 2010 in neighbouring countries (see below). They were found to represent a monophyletic group most closely related to the viruses isolated in Uttar Pradesh (India) from two polio cases with disease onset on 1 October and 6 November 2009 (Figure 3). Phylogenetic analysis suggested that the outbreak in Tajikistan resulted from a single importation event. Consistent with a rapid development of the outbreak, the majority of the isolates exhibited a low level of genetic divergence and did not cluster into distinct, statistically supported lineages, although the maximum VP1 nucleotide divergence between the WPV 1 isolated in Tajikistan was $1.7 \%$.

The VP1 diversity between the most diverged members of this monophyletic population, RUS38492 (Dagestan, 18 September 2010) and RUS38655 (Chechnya, 6 October 2010) was $2.3 \%$. On the basis of the phylogenetic reconstruction, it may be estimated that these viruses had independently evolved from a common ancestor ca 14 months earlier (with 95\% highest probability density confidence interval (HPD) of $12.3-15.8$ months) (Figure 3). The mean rate of nucleotide substitutions comprised ca 1.28\% (95\% HPD: 1.05-1.51\%) per 


\section{FIgURE 3}

Maximum clade credibility tree for the VP1 coding region of wild polioviruses isolated during the outbreak in Tajikistan and neighbouring countries, $2010\left(\mathrm{n}=119^{\mathrm{a}}\right)$ and of isolates circulating in Uttar Pradesh in $2008(\mathrm{n}=5)$ and 2009 $(\mathrm{n}=17)$

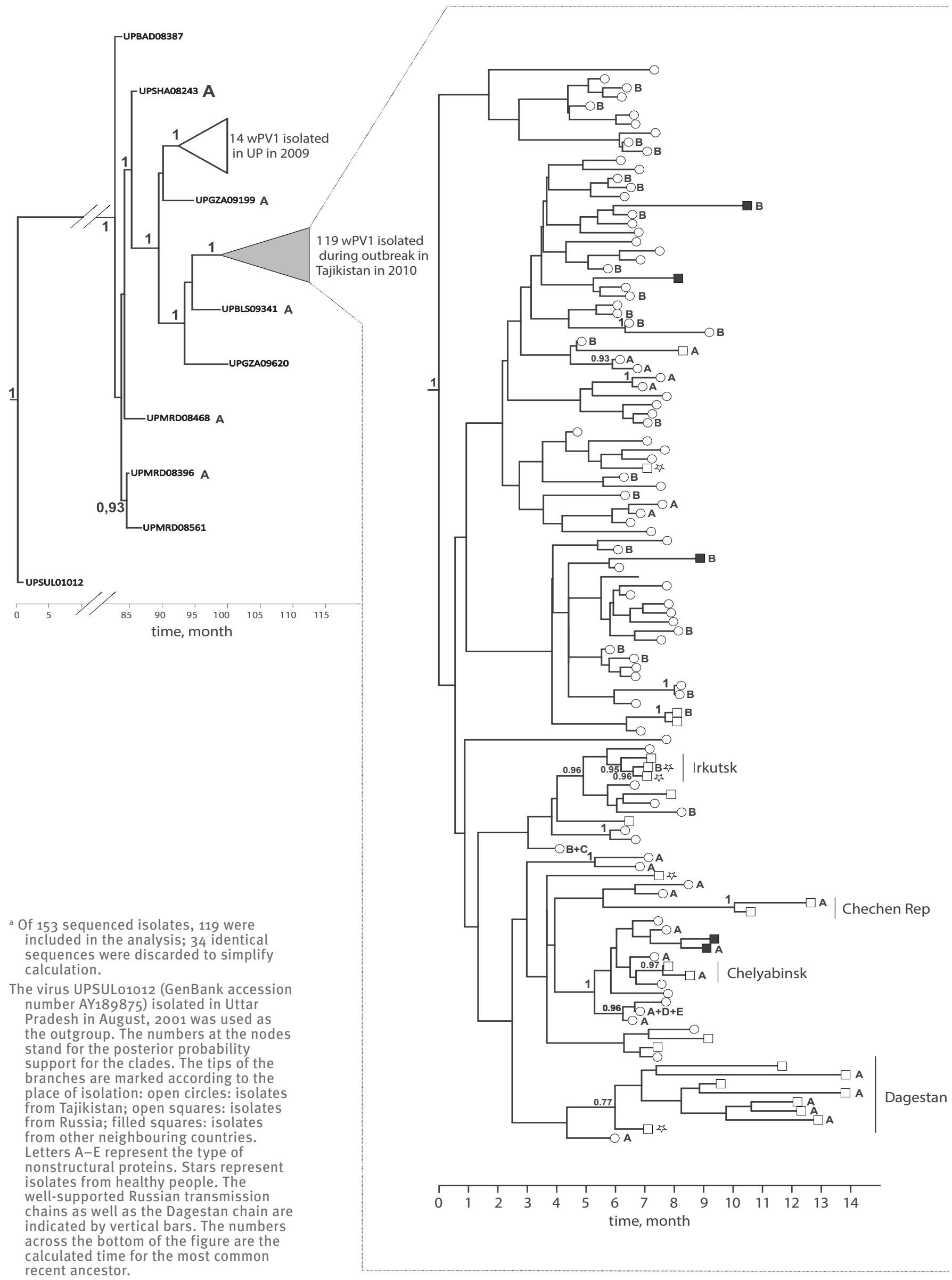


FIGURE 4

Recombinant genomes of polioviruses isolated during the outbreak in Tajikistan and neighbouring countries, 2010

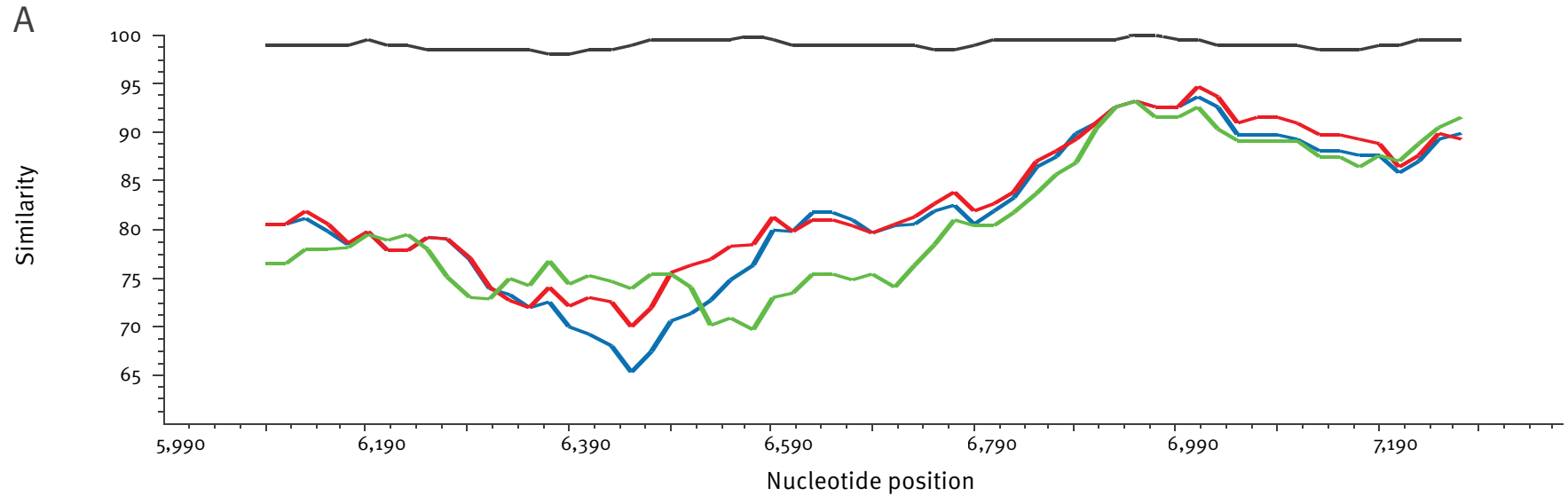

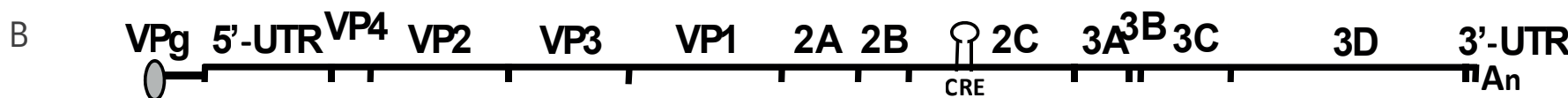
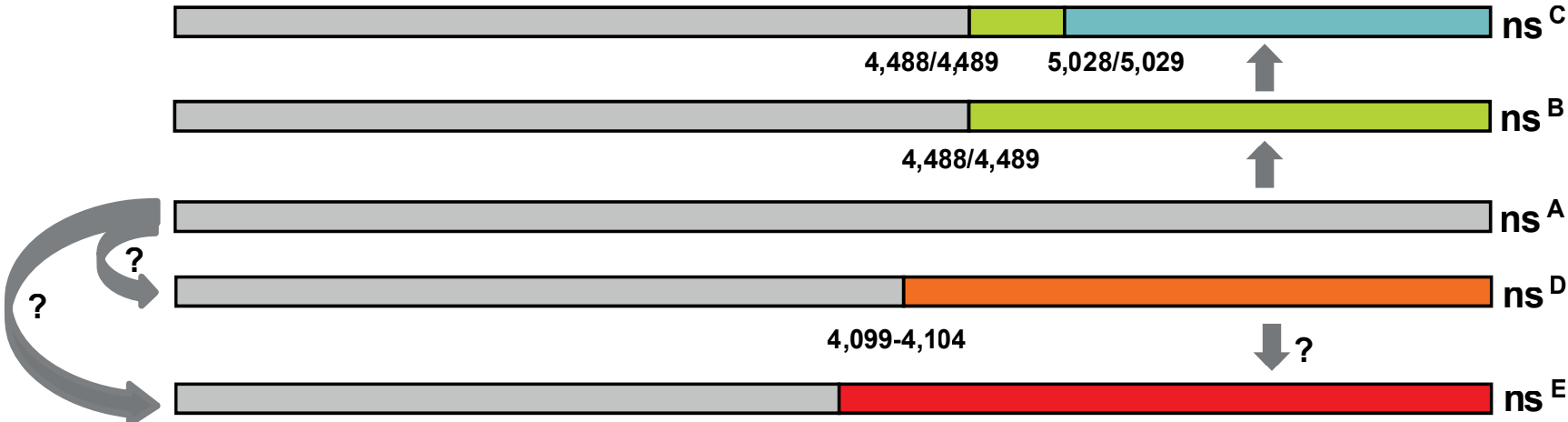

$3,754 / 3,755$

C

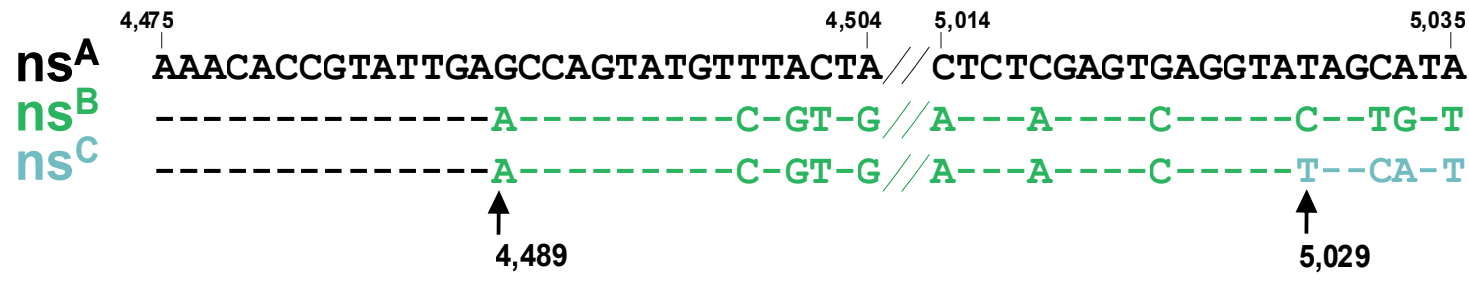

D $\quad 3,744 \quad 3,769 \quad 4,093 \quad 4,128$

ns $^{A}$ TTATAGGCATAGTCACTGCAGGAGGG//GACATTCTGGAGATCCCATATGTCATTCGTCAAGGA

nS ${ }^{\mathrm{D}}--------------------------/ /-----------A-----------\mathrm{G}-----\mathrm{C}-----\mathrm{T}$

$\mathrm{nS}^{\mathrm{E}}----------\mathrm{A}----------\mathrm{G}--\mathrm{T} / /-----\mathrm{A}-----\mathrm{A}-----------\mathrm{A}-----\mathrm{C}-----\mathrm{T}$

$3,755 \quad 4,099-4,104$

ns: non-structural; UTR: untranslated region.

(A) Similarity plots of a1,383 nt long genome segment (position 5,990-7,372) of $\mathrm{ns}^{\mathrm{A}}$ (gray), $\mathrm{ns}^{\mathrm{B}}$ (green), $\mathrm{ns}^{\mathrm{C}}(\mathrm{blue}), \mathrm{gs}^{\mathrm{D}}$ and $\mathrm{ns}^{\mathrm{E}}$ (red) isolates compared with the sequence of wild poliovirus type 1 UPBSL09341 isolated in Uttar Pradesh in 2009. The nsA variant was inherited from an Indian predecessor, while other variants appeared to originate in Tajikistan.

(B) Schematic representation of five types of the non-structural genomic region. Possible relations between different variants are indicated by arrows.

(C) Nucleotide sequences in the proximity of the crossover points of $n s^{B}$ and $n s^{C}$ recombinants compared with the parental $n s^{A}$ sequence.

(D) Nucleotide sequences in the proximity of crossover points of $n s^{D}$ and $n s^{E}$ recombinants compared with the parental $n s^{A}$ sequence. Identical nucleotides are marked by dashes. 
Population immunity to polioviruses in Tajikistan, $2010(n=327)$
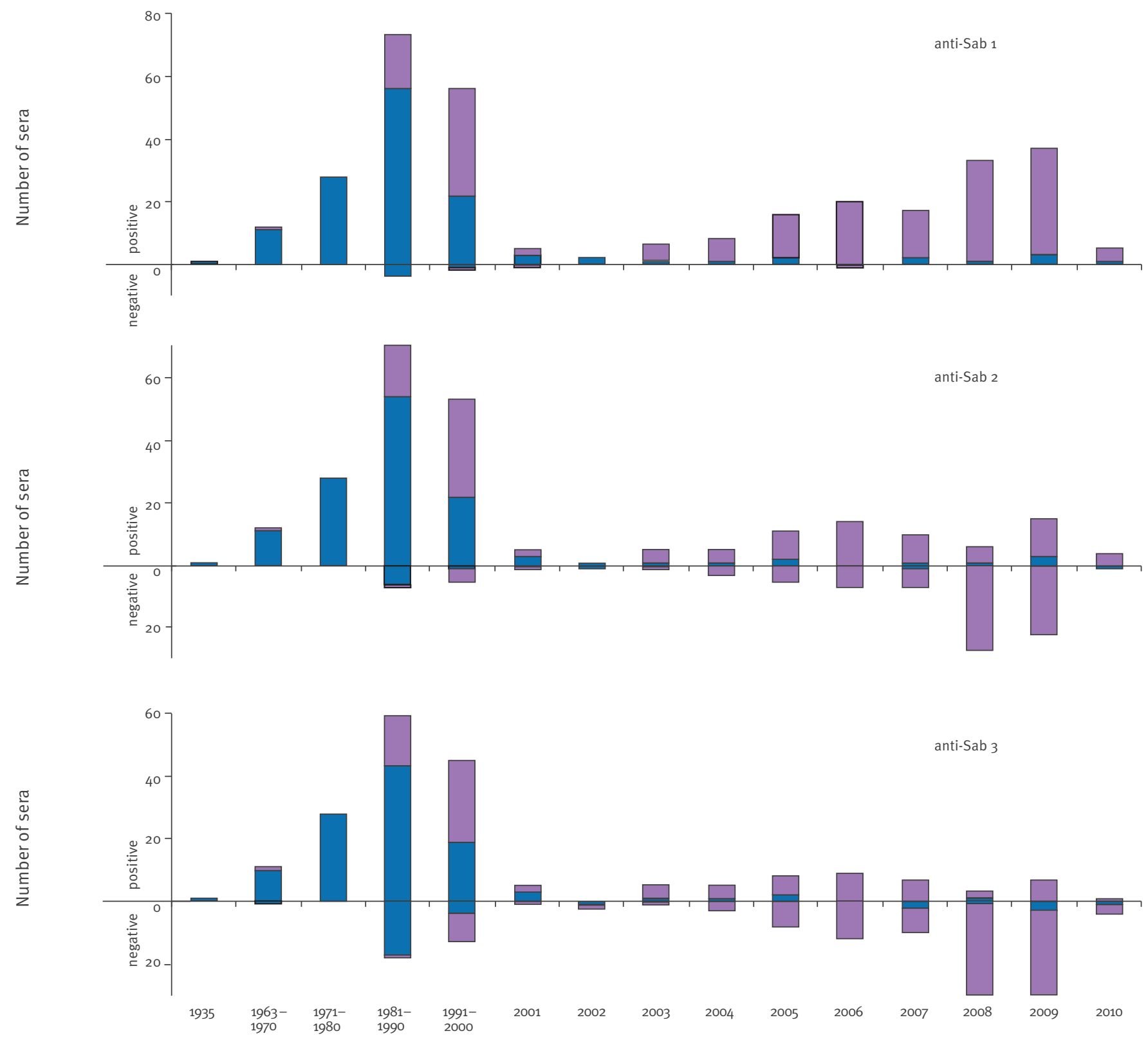

Birth year

Sera collected from patients with AFP (blue bars) as well as from healthy people or patients with other diagnoses (purple bars) were tested in microneutralisation assay with type 1, type 2, and type 3 Sabin strains (panels presented as anti-Sab1, anti-Sab2, anti-Sab3). Year of birth of sera donors is indicated below the corresponding bars. Positive and negative sera correspond to samples containing $\geq 1: 8$ and $<1: 8$ titres of neutralising antibodies respectively. 
year, i.e. close to the earlier estimates by Gavrilin et al [15] and Jorba et al [16].

Nucleotide variability in the VP1 coding region mostly affected synonymous sites (147 synonymous substitutions per 182 changed positions). The majority of nonsynonymous mutations were sporadic and only one, Glutamine to Arginine at position VP1-004 (the amino acid residues are numbered starting at VP1-001) was observed in 12 of 153 analysed WPV 1 sequences. This and several other mutations, which mapped to, or close to, antigenic sites and/or sites involved in interaction of poliovirus with its receptor, did not deviate from the VP1 consensus for WPV deposited in the GenBank and appeared to reflect 'permitted' variability with little or no adaptive significance.

\section{Whole genome sequencing}

Full genome sequences of 14 WPV1 isolates from Tajikistan $(n=6)$, Russia $(n=6)$, Kazakhstan $(n=1)$ and Turkmenistan $(n=1)$, were determined. Several amino acid residues of the capsid proteins other than VP1 were found to be unique (not present in the GenBank) and two of them appeared to map to the antigenic/ receptor-interacting regions. However, neither of them was consistently fixed in a significant number of isolates or reflected a substantial change in the physicalchemical properties of the residues, again suggesting that this variability was of a non-adaptive nature.

These sequences as well as partial sequences of the non-structural region of 37 additional isolates ( 32 from Tajikistan, four from Russia and one from Turkmenistan) revealed, in addition to mutations, several recombination events. Comparative analysis indicated that one set of nonstructural proteins (designated $\mathrm{ns}^{\mathrm{A}}$ ) was inherited from the parental Indian strain (Figures 3 and 4 ), whereas another $\left(n s^{B}\right)$ appeared to be acquired by recombination with an unknown enterovirus C (Figure
4) before February 2010, because the very first WPV 1 isolate in Tajikistan (the case dated 1 February 2010) already was of $n s^{B}$ type. Since that isolate exhibited sequence heterogeneity, plaque cloning was performed. As a result, an additional related recombinant was identified. The crossover region in the $\mathrm{ns}^{\mathrm{B}}$-harbouring isolate mapped to around nt position 4,488 in the $2 \mathrm{C}$ region, whereas the other isolate $\left(\mathrm{ns}^{\mathrm{C}}\right)$, while sharing ca $540 \mathrm{nt}$ with that of $\mathrm{ns}^{\mathrm{B}}$, had acquired the sequence downstream of position 5,028 from another enterovirus C (Figure 4). Detection of the $\mathrm{ns}^{\mathrm{B}}$ set of proteins in numerous WPV 1 isolates (Figure 3) suggested widespread co-circulation of these viruses together with $\mathrm{ns}^{\mathrm{A}}$-harbouring viruses. On the other hand, $\mathrm{ns}^{\mathrm{C}}$ proteins were detected only in the isolate from a single patient and thus appeared to represent a dead-end population (although it should be admitted that only a limited proportion of isolates $(n=51)$ were sequenced in the non-structural region and all isolates were from cases of poliomyelitis).

Sequencing of a WPV 1 isolate from another patient also revealed heterogeneity. Cloned populations of this isolate demonstrated the presence, along with the $\mathrm{ns}^{\mathrm{A}}$-containing virus, of two recombinants possessing distinct, though closely related, sets of non-structural proteins, $n s^{D}$ and $n s^{E}$. The crossing partner(s) of the $\mathrm{ns}^{\mathrm{A}}$-containing virus appeared to be another human enterovirus C (or two related viruses), which donated their sequences downstream of nt positions 3,755 and 4,104 , respectively (Figure 4). It is unclear whether there were in this case two independent recombination events, or one recombinant was the predecessor of the other (Figure $4 \mathrm{~B}$ ). Neither $n s^{\mathrm{D}}$ nor $n s^{\mathrm{E}}$ proteins were detected in any other sequenced isolate. Although numerous mutations in the non-structural proteins were detected, we were unable to ascribe to any of them an obvious biological significance.

\section{TABLE 1}

Antibodies in sera of poliovirus-negative cases of acute flaccid paralysis registered before the start of the supplementary immunisation activities, Tajikistan, $2010(n=8)$

\begin{tabular}{|l|c|c|c|c|c|}
\hline \multirow{2}{*}{ Year of birth } & \multicolumn{3}{|c|}{ Microneutralisation } & \multicolumn{2}{c|}{ ELISA (Sab1) } \\
\cline { 2 - 6 } & anti-Sab1 & anti-Sab2 & anti-Sab3 & $1 \mathrm{IgM}$ & 25,600 \\
\hline $1986^{\mathrm{a}}$ & 841 & 1,024 & 11,024 & 1,600 & 6,400 \\
\hline $1990^{\mathrm{a}}$ & 299 & 251 & 1180 & 3,200 \\
\hline $1994^{\mathrm{a}}$ & 1,024 & 1,024 & 421 & 100 & 25,600 \\
\hline $1996^{\mathrm{b}}$ & $>1,024$ & $>1,024$ & $<8$ & 100 & 100 \\
\hline $2006^{\mathrm{b}}$ & $<8$ & $<8$ & $<8$ & 12,800 & 3,200 \\
\hline $2008^{\mathrm{a}}$ & 299 & $<8$ & $<8$ & 400 & 3,200 \\
\hline $2008^{\mathrm{b}}$ & 299 & 708 & $<8$ & 1,600 & 6,400 \\
\hline $2010^{\mathrm{c}}$ & 708 & 8 & & \\
\hline
\end{tabular}

ELISA: enzyme-linked immunosorbent assay; Sab: Sabin vaccine strain.

\footnotetext{
a Data on the vaccination not available.

b Patients reported to be vaccinated before 2010 .

c Patient reported to be vaccinated late in March, 2010 before the SIAs were started.
} 
The observed variability in the 5'- and $3^{\prime}$-untranslated regions did not appear to affect the stability of their secondary structures (not shown).

\section{Conditions favouring the outbreak}

To provide insight into conditions favouring the outbreak, 327 sera obtained before and during the outbreak from 188 randomly selected patients with AFP (144 laboratory-confirmed, 42 WPV-negative and two not investigated) and 139 healthy persons or patients with other diagnoses were assayed for poliovirus antibodies. A low immunity to poliovirus types 2 and 3 was revealed in 67 of 137 children born in 2004 to 2010 (Figure 5). Neutralising antibodies to these serotypes were not detected ( $11: 8)$ in $37 \%$ of the sera from all AFP cases and in $64 \%$ sera of 81 AFP patients born in 2007 to 2009, testifying to insufficient population immunity, which is very likely to have contributed to the outbreak onset and size.

\section{Additional serological features}

Of the eight poliovirus-negative patients who developed AFP before the supplementary immunisation activities and from whom sera were collected, at least three seemed to be unvaccinated as they lacked neutralising antibodies to Sabin strains of serotypes 2 and 3. Two of them exhibited high titres of both neutralising antibodies and IgM to poliovirus type 1 (Table 1), consistent with a recent infection with this virus. The data from four patients raised the possibility that even

\section{TABLE 2}

High levels of neutralising antibodies in the laboratoryconfirmed poliomyelitis cases registered before the start of the supplementary immunisation activities ${ }^{\mathrm{a}}$, Tajikistan, $2010(n=13)$

\begin{tabular}{|c|c|c|c|}
\hline \multirow{2}{*}{ Year of birth } & \multicolumn{3}{|c|}{ Microneutralisation } \\
\cline { 2 - 4 } & anti-Sab1 & anti-Sab2 & anti-Sab3 \\
\hline $1990^{\mathrm{b}}$ & 1,024 & 355 & 53 \\
\hline $1995^{\mathrm{c}}$ & 708 & 708 & 32 \\
\hline $1996^{\mathrm{c}}$ & 596 & 355 & 299 \\
\hline $2003^{\mathrm{b}}$ & 251 & 501 & 211 \\
\hline $2003^{\mathrm{b}}$ & 150 & 355 & 1,024 \\
\hline $2003^{\mathrm{b}}$ & 1,024 & 1,024 & 251 \\
\hline $2005^{\mathrm{c}}$ & 421 & 1,024 & 75 \\
\hline $2005^{\mathrm{c}}$ & 1,024 & 501 & 45 \\
\hline $2006^{\mathrm{b}}$ & 75 & 32 & 1,024 \\
\hline $2006^{\mathrm{b}}$ & 150 & 45 & 422 \\
\hline $2006^{\mathrm{b}}$ & 501 & 1,024 & 501 \\
\hline $2007^{\mathrm{b}}$ & 89 & 502 & 75 \\
\hline $2009^{\mathrm{b}}$ & 422 & 1,024 & 211 \\
\hline
\end{tabular}

Sab: Sabin vaccine strain.

Only data for sera with high titres $(\geq 1: 32)$ of neutralising antibodies to the three poliovirus serotypes are included.

Data on the vaccination not available.

Patients vaccinated before 2010. poliovirus-negative AFP patients who possessed neutralising antibodies to all poliovirus serotypes and thus were seemingly effectively vaccinated, might present paralytic disease. Indeed, the sera of one such case had a very high IgM titre against poliovirus 1 , suggestive of acute infection. Although non-polio AFP aetiology in some of these cases cannot be rigorously excluded, their appearance during the poliomyelitis outbreak suggests that they were more likely to be caused by WPV1. Remarkably, 13 of the 144 patients with laboratory-confirmed poliomyelitis were serotypepositive against all the three polio serotypes (Table 2) and thus were also likely to be vaccinated.

\section{Export to neighbouring countries}

During the outbreak, WPV1 was exported from Tajikistan to several neighbouring countries, Kazakhstan, Russia, Turkmenistan and Uzbekistan. The largest number of disease cases caused by this virus, 19 cases of paralytic poliomyelitis, was observed in Russia (Figure $2 B$ ). Seven of these patients had recently come from Tajikistan or Uzbekistan or had known contacts in these countries. Fifteen cases were registered in Russian citizens, predominantly in the North Caucasus region (seven cases in Dagestan and four in Chechnya). The age of the 19 poliomyelitis patients ranged from six months to 32 years, and eight of them had no antibodies against poliovirus types 2 and 3 and were therefore likely to be unvaccinated. Of note, among patients in Dagestan, three were adults (19, 27 and 32 years-old); two of them may have been vaccinated, judging by the presence of antibodies to polioviruses types 2 and 3 . WPV 1 was also isolated from 39 of 438 investigated healthy contacts, including four persons between 48 and 80 years-old.

Sequencing data indicate that all the isolates from Russia were closely related to the strains circulating in Tajikistan and that there were at least 10 independent virus importations (Figure 3). In some cases, the transmission route from Tajikistan to Russia could be established with reasonable certainty. Thus, the virus with Arg VP1-004 was isolated in adjacent districts of Tajikistan starting from February 2010, and viruses with this marker were later, from May 2010, isolated in Russia as well as in Uzbekistan. The non-structural genomic regions of three of 12 isolates possessing this marker were sequenced and found to share non-structural proteins of $n s^{B}$ type.

Several chains of WPV 1 transmission within Russia could also be identified (Figure 3). One was detected in the Irkutsk Region, with the first WPV 1 isolated on 4 May from an AFP-diagnosed patient recently arrived from Tajikistan, the second from a healthy contact on 6 May, and the third from an unrelated healthy person on 9 June. A second transmission chain was due to importation to the Chelyabinsk Region around the end of April. Two cases were registered, on 10 May and 2 June, suggesting circulation of the virus for at least three weeks. The importation resulting in the transmission 
chain in Chechnya, with onset of the first two polio cases on 4 August (estimated importation date on around 11 July), appeared to occur after the last case of WPV1-caused poliomyelitis was registered in Tajikistan on 4 July. Two subsequent isolations from poliomyelitis victims were on 10 August and 6 October 2010, suggesting circulation for over two months. One more transmission chain may have occurred in Dagestan, where seven polio cases were registered from 15 July to 22 November 2010. However, due to a relatively weak statistical support (0.77), several independent importations to Dagestan cannot be rigorously excluded.

Several WPV1 were isolated from faecal samples collected in 2010 from AFP cases in other neighbouring countries, two in Uzbekistan (six months- and 11 yearsold, both vaccinated; dates of onset in April and May), three in Turkmenistan (two-, 11 and 13 years-old, all vaccinated; dates of onset in June), and one in Kazakhstan (seven years-old, vaccinated; date of onset in August). All of them were closely related to the Tajikistan isolates (Figure 3).

\section{Discussion}

The viruses isolated during the Tajikistan poliomyelitis outbreak of $\mathbf{2 0 1 0}$ were closely related to the strains that circulated in the Indian state of Uttar Pradesh between 1 October and 6 November 2009. A single importation of WPV 1 appears to have occurred in October 2009 (mean date with 95\% HPD: end of August-early December) (Figure 3), i.e. well before the first isolation of WPV 1 in Tajikistan, and the lag between this importation and first detection of the virus in Tajikistan could be explained by a low case:infection ratio for WPV 1 infections and/or inadequate AFP surveillance. It may be noted that movement of people exists between the two countries, and this or a similar route of the crossborder poliovirus transmission was recorded also in 1991, when a strain of WPV1 circulating in Tajikistan (so called T-geotype) was found to be closely related to viruses found during the same period in India and Pakistan $[15,17]$. It may be noted that the VP1 sequences of the T-geotype and the contemporary viruses isolated in India and Tajikistan exhibited only $13-15 \%$ similarity. Moreover, the Tajikistan outbreak is a recent example of a long-standing process of importation of WPV from South Asia to Central and East Asia. Other examples include importation into Xinjiang, China in 2011 [18] and in 1999 into Qinghai, China [19].

\section{The size of the outbreak}

The number of AFP cases registered in Tajikistan.during the outbreak was 715 , of whom 463 were diagnosed as poliomyelitis cases and 47 as polio-compatible cases; 181 WPV-negative patients and 24 patients without adequate faecal samples were classified as non-polio AFP cases. However, there is reason to believe that in reality the outbreak in Tajikistan in $\mathbf{2 0 1 0}$ was larger.

Indeed, the number of AFP cases with unavailable or inadequate faecal specimens $(n=71$ cases) or
WPV1-negative specimens $(n=181)$ was very high. The reported mean yearly AFP incidence among children under the age of 15 years from 2000 to 2009.in Tajikistan was $30 \pm 8$ [20], which corresponds to a rate of slightly above one case of AFP per 100,000 children under 15 years of age. The sharp, ca six-fold, increase in the number of non-polio AFP cases in 2010 was unusual. It is plausible that a proportion of them were associated with WPV1 infection. It is noteworthy that large areas in Tajikistan in 2010 had a significant number of AFP cases with no confirmed poliomyelitis (Figure 2A).

A significant number of poliovirus-negative AFP cases in the context of the 2010 outbreak were classified by the National Expert Committee as polyneuropathy and Guillain-Barré syndrome. However, in-depth clinical investigations such as the cerebrospinal fluid analysis, electromyography and nerve conductance studies had not been performed. Our own clinical observations (data not shown) suggest that at least two lethal AFP cases with unavailable or inadequate faecal specimens were discarded by the National Expert Committee but could, according to the current criteria, be regarded as polio-compatible. The presence of neutralising antibodies and high-titre IgM to type 1 poliovirus in the absence of neutralising antibodies to other poliovirus serotypes in two of the three apparently poliovirusnegative AFP cases may also be considered as an indication for likely recent infection with WPV1.

It cannot be excluded that the unusually high prevalence of AFP cases in 2010 may be partly explained by overreporting during the outbreak or underreporting in the previous years. Nevertheless, we think the available data are largely representative, even if not sampled systematically. If so, this would testify to limitations of the AFP incidence statistics as a key indicator in the current polio surveillance, at least in certain areas.

It is highly surprising that no polio cases were reported from Uzbekistan, as cases occurred across the border in Tajikistan (Figure 2A) and cross-border travel is frequent. Moreover, one patient developed paralysis (and excreted poliovirus closely related to the Tajikistan WPV1) one day after arrival to Moscow from Uzbekistan in the beginning of June, 2010 [21]. WPV 1 were also detected in faecal samples from two AFP cases in Uzbekistan.

It may be added that paralytic diseases are known to be presented only by a minority of the infected persons, e.g. one case per 190 for WPV 1 [22], suggesting that there may have been tens of thousands infections in Tajikistan in 2010.

\section{Conditions facilitating the outbreak}

A marked proportion of AFP victims of the Tajikistan outbreak who were born between 2004 and 2010, had no detectable antibodies to poliovirus serotypes 2 and 3 and hence were most probably not vaccinated. A serological survey conducted by Khetsuriani et al. after 
the start of vaccination with mOPV and before tOPV implementation also showed a low level of less than 90\% population immunity to type 3 poliovirus [23].

Although observations made in India demonstrate that vaccination may fail to induce adequate antibody response under certain conditions [24], the situation in Tajikistan appears to be different, judging by the effectiveness of vaccinations conducted to curb the outbreak (Figure $1 \mathrm{~A}$ ). The estimated anti-polio vaccination coverage with three doses of OPV in Tajikistan in 2005 to 2008 varied between 76 and $87 \%$ [25]. Thus, the inadequate level of immunity before the outbreak appears to have been a consequence of failure to vaccinate rather than of vaccine failure. It may be noted, that many countries, including several European ones, demonstrate similar low, or even lower, polio vaccination coverage [26], a situation requiring urgent measures to diminish the risk of similar outbreaks.

Special attention deserves the circulation of poliovirus among teenagers older than 15 years and adults. This age cohort is rarely targeted by vaccination campaigns, but can play a significant part in transmission of the virus.

Paralytic disease in previously vaccinated people Although the major factor favouring the development of this outbreak was inadequate population immunity against poliovirus, there is evidence, although circumstantial, that paralytic poliomyelitis may on extremely rare occasions occur in OPV-vaccinated people who have a sufficient level of appropriate antibodies (Table 2). This observation by no means discredits the efficacy or safety of OPV but should be taken into account during epidemiological analyses. It may be added that the possibility of polio infection (without paralytic manifestations) of vaccinated people has been reported previously, e.g. in people with deficient mucosal immunity $[27,28]$.

\section{Epidemiological relevance of recombinants} between wild polioviruses and other enteroviruses

Recombination between WPV or vaccine-derived polioviruses with other enteroviruses $C$ is well known [29-31], but its biological relevance remains unclear. Recombination between WPV1 and several human enteroviruses C took place in Tajikistan in 2010. Interestingly, only one of several detected recombinants with newly acquired nonstructural proteins $\left(\mathrm{ns}^{\mathrm{B}}\right)$ appeared to cocirculate with the originally imported lineage. It could be speculated that the failure of other recombinants to circulate was due to poor mutual compatibility of their genes. Another possibility is that at least some of these other recombinants did circulate but did not cause neurological disease and therefore could not be detected in samples from AFP cases. It should also be kept in mind that non-structural regions were analysed in only a restricted set of isolates.
Do those recombinants that are sufficiently fit (i.e. able to spread in human populations) have any advantage compared to the non-recombinants? An interesting, if speculative, possibility could be a role for nonstructural poliovirus proteins in eliciting an immune response. This postulate is partially supported by the detection of antibodies against poliovirus non-structural proteins in some sera of poliovirus-positive AFP cases (data not shown; see also [32]). It is of note that non-structural proteins of other picornaviruses such as foot-and-mouth disease virus [33,34], hepatitis $A$ virus [35], and Theiler's murine encephalomyelitis virus $[36,37]$ do serve as targets for antiviral immunity. If this reasoning is valid, exchanges of non-structural proteins may facilitate immune evasion. We were, however, not able to demonstrate the ability of sera from poliomyelitis patients to discriminate between viruses with different sets of non-structural proteins $\left(\mathrm{ns}^{\mathrm{A}}\right.$ and $n s^{B}$ ). This hypothesis deserves further testing.

\section{Conclusion}

Several important lessons can be learnt from the analysis of the outbreak in Tajikistan. The main circumstance permitting the spread of the imported WPV was low population immunity against poliovirus. The effectiveness of the vaccination in stopping the outbreak indicates that there were no factors strongly interfering with the development of immune response against poliovirus and that people with no protective antibodies were most likely not to be (correctly) vaccinated. Serological surveillance can provide objective measures independent of official estimates of polio vaccination coverage. This surveillance should not neglect the immunological status of adults.

This outbreak, as well as an outbreak in the Republic of Congo in 2010 [38], highlight the need for continuous vaccination against poliomyelitis [39], especially taking into account a low level of population immunity against poliovirus in numerous countries. Moreover, the currently adopted vaccination strategy, i.e. a gradual switch from OPV to IPV [40], may need to be reconsidered, particularly in circumstances such as the recent silent transmission of $W P_{1}$ in Israel [41]. The possibility of circulation of WPV should be taken into account by the health authorities of the countries that use an IPV-only schedule. Finding the optimal way for maintaining adequate population immunity to poliovirus is an urgent challenge.

\section{Acknowledgments}

The study was conducted with financial support of the World Health Organization, the Russian Foundation for Basic Research of the Russian Ministry of Education and Science and the Government of Russian Federation. We would like to thank Ministry of Health and State Center of Immunoprophylaxis of the Republic of Tajikistan for providing the outbreak specimens and epidemiological information to Moscow Polio Regional Reference Laboratory of WHO, and F. Tishkova, Tajik Scientific Research Institute of Preventive 
Medicine, for providing a collection of sera samples; Federal Service for Surveillance on Consumer Rights Protection and Human Wellbeing (Rospotrebnadzor), Federal Center for Epidemiological Surveillance, and Polio laboratory network of Russian Federation for assistance in collection and delivery of the clinical specimens and providing epidemiological data; I. Gordeychuk for help with full-genome sequencing of some viral clones, A. N. Lukashev and A. E. Gorbalenya for advice; A.Goel for assistance with clarification of some epidemiological data and M. Kolesnikova for technical support.

\section{Conflict of interest}

None declared.

\section{Authors' contributions}

MLY, APG, OEI, VIA - planned the study, MLY, APG, MAP, OVI, JMD - performed molecular analyses, OEI, TPE, OYB performed initial laboratory analysis of fecal samples, API - performed analyses of sera samples, AKS - conducted clinical investigation of polio cases in Tajikistan at the beginning of the outbreak as a member of expert group, MLY, APG, OEI, TPE, TPE, API, GYL, AKS, OKM, JMD, VIA - discussed the results, MLY, APG, VIA drafted the manuscript, all co-authors reviewed the final version of the manuscript.

\section{References}

1. Forty-first World Health Assembly. Global eradication of poliomyelitis by the year 2000. Resolution WHA 41.28. Geneva: World Health Organization; 1988. Available from: http:// www.polioeradication.org/content/publications/19880513 resolution.pdf

2. Global Polio Eradication Initiative. Wild Poliovirus 2009 2014. Geneva: World Health Organization; 2014. Available from: http://www.polioeradication.org/Portals/o/Document/ Data\&Monitoring/Wild_poliovirus_list_2008_2014_11Feb.pdf

3. Agol VI.Vaccine-derived polioviruses. Biologicals. 2006;34(2):103-8. http://dx.doi.org/10.1016/j. biologicals.2006.02.007

4. Centers for Disease Control and Prevention. Outbreaks following wild poliovirus importations - Europe, Africa, and Asia, January 2009 - September 2010. MMWR Morb Mortal Wkly Rep. 2010;59(43):1393-9.

5. World Health Organization Regional Office for Europe (WHO/ Europe). Report of the 24th Meeting of the European Regional Certification Commission for Poliomyelitis Eradication. Copenhagen: WHO/Europe; 2011. Available from: http://www. euro.who.int/_data/assets/pdf_file/0018/145314/e95119.pdf

6. World Health Organization (WHO). Manual for the virological investigation of poliomyelitis. 4th ed. WHO/IVB/04.10. Geneva: WHO; 2004. Available from: http://whqlibdoc.who.int/ hq/2004/WHO_IVB_04.10.pdf

7. van der Avoort HG, Hull BP, Hovi T, Pallansch MA, Kew OM, Crainic R, et al. A comparative study of five methods of intratypic differentiation of polioviruses. J ClinMicrobiol. 1995;33(10):2562-6.

8. Kilpatrick DR, Yang CF, Ching K, Vincent A, Iber J, Campagnoli $R$, et al. Rapid group-, serotype-, and vaccine strainspecific identification of poliovirus isolates by real-time reverse transcription-PCR using degenerate primers and probes containing deoxyinosine residues. J Clin Microbiol. 2009;47(6):1939-41. http://dx.doi.org/10.1128/JCM.00702-09

9. Kilpatrick DR, Iber JC, Chen Q, Ching K, Yang SJ, De L, et al. Poliovirus serotype-specific VP1 sequencing primers. J Viro Methods. 2011;174(1-2):128-30. http://dx.doi.org/10.1016/j. jviromet.2011.03.020

10. World Health Organization (WHO). Manual for the virological investigation of polio. WHO/EPI/GEN/97.1.1. Geneva: WHO; 1997. Available from: http://whqlibdoc.who.int/hq/1997/ WHO_EPI_GEN_97.01.pdf

11. Dragunsky EM, Ivanov AP, Wells VR, Ivshina AV, Rezapkin GV, Abe $S$, et al. Evaluation of immunogenicity and protective properties of inactivated poliovirus vaccine: a new surrogate method for predicting vaccine efficacy. J Infect Dis. 2004;190(8):1404-12. http://dx.doi.org/10.1086/424524
12. Yakovenko ML, Cherkasova EA, Rezapkin GV, Ivanova OE, Ivanov AP, Eremeeva TP, et al. Antigenic evolution of vaccinederived polioviruses: changes in individual epitopes and relative stability of the overall immunological properties. J Virol. 2006;80(6):2641-53. http://dx.doi.org/10.1128/ JVI.80.6.2641-2653.2006

13. Drummond AJ, Suchard MA, Xie D, Rambaut A. Bayesian phylogenetics with BEAUti and the BEAST 1.7. Mol Biol Evol. 2012;29(8):1969-73. http://dx.doi.org/10.1093/molbev/mss075

14. Lole KS, Bollinger RC, Paranjape RS, Gadkari D, Kulkarni SS, Novak NG, et al. Full-length human immunodeficiency virus type 1 genomes from subtype C-infected seroconverters in India, with evidence of intersubtype recombination. J Virol. 1999;73(1):152-60.

15. Gavrilin GV, Cherkasova EA, Lipskaya GY, Kew OM, Agol VI. Evolution of circulating wild poliovirus and of vaccine-derived poliovirus in an immunodeficient patient: a unifying model. j Virol. 2000;74(16):7381-90. http://dx.doi.org/10.1128/ JVI.74.16.7381-7390.2000

16. Jorba J, Campagnoli R, De L, Kew O. Calibration of multiple poliovirus molecular clocks covering an extended evolutionary range. J Virol. 2008;82(9):4429-40. http://dx.doi.org/10.1128/ JVI.02354-07

17. Lipskaya GY, Chervonskaya EA, Belova GI, Maslova SV, Kutateladze TN, Drozdov SG, et al. Geographical genotypes (geotypes) of poliovirus case isolates from the former Soviet Union: relatedness to other known poliovirus genotypes. J Gen Virol. 1995;76(Pt 7):1687-99. http://dx.doi. org/10.1099/0022-1317-76-7-1687

18. World Health Organization Regional Office for the Western Pacific. Wild poliovirus in China. WHO/Western Pacific. [Accessed: 18 February 2014]. Available from: http://www. wpro.who.int/immunization/news/wild_poliovirus_China/en/ index.html

19. Chiba Y, Murakami H, Kobayashi M, Shimizu H, Yoshida H, Yoneyama T, et al. A case of poliomyelitis associated with infection of wild poliovirus in Qinghai Province, China, in October 1999. Jpn J Infect Dis. 2000;53(3):135-6.

20. Global Polio Eradication Initiative. Surveillance. Geneva. World Health Organization. [Accessed: 18 February 2014]. Available from: http://www.polioeradication.org/Dataandmonitoring/ Surveillance.aspx

21. Shyshov AS, Saifullin MA, Shakaryan AK, Ivanova OE, Sachkova IY. [A case of poliovirus infection caused by a wild strain in the adult patient.] Zh Nevrol Psikhiatr Im S S Korsakova. 2011;111(10 Pt 1):77-80. Russian.

22. Nathanson N, Kew OM. From emergence to eradication: the epidemiology of poliomyelitis deconstructed. Am J Epidemiol. 2010;172(11):1213-29. http://dx.doi.org/10.1093/aje/kwq320

23. Khetsuriani N, Pallansch MA, Jabirov S, Saparova N, Oberste MS, Wannemuehler K, et al. Population immunity to polioviruses in the context of a large-scale wild poliovirus type 1 outbreak in Tajikistan, 2010. Vaccine. 2013;31(42)4911-6. http://dx.doi.org/10.1016/j.vaccine.2013.06.106

24. Grassly NC, Fraser C, Wenger J, Deshpande JM, Sutter RW, Heymann DL, et al. New strategies for the elimination of polio from India. Science. 2006;314(5802):1150-3. http://dx.doi. org/10.1126/science.1130388

25. World Health Organization; United Nations Children's Fund. Countries and territories, Tajikistan. In: Immunization summary. A statistical reference containing data through 2012, WHO/UNICEF; 2014. p 171. Available from: http://www. childinfo.org/files/immunization_summary_2012_en.pdf

26. World Health Organization (WHO). WHO-UNICEF estimates of Pol3 coverage. Geneva: WHO. [Accessed:18 February 2014]. Available from: http://apps.who.int/immunization_monitoring/ globalsummary/timeseries/tswucoveragepol3.html

27. Grassly NC, Jafari H, Bahl S, Durrani S, Wenger J, Sutter RW, et al. Asymptomatic wild-type poliovirus infection in India among children with previous oral poliovirus vaccination. J Infect Dis 2010;201(10):1535-43.http://dx.doi.org/10.1086/651952

28. Ogra PL, Okayasu H, Czerkinsky C, Sutter RW. Mucosal immunity to poliovirus. Expert Rev Vaccines. 2011;10(10):138992. http://dx.doi.org/10.1586/erv.11.106

29. Dahourou G, Guillot S, Le Gall O, Crainic R. Genetic recombination in wild-type poliovirus. J Gen Virol. 2002;83(Pt 12):3103-10.

30. Liu HM, Zheng DP, Zhang LB, Oberste MS, Kew OM, Pallansch MA. Serial recombination during circulation of type 1 wild-vaccine recombinant polioviruses in China. J Virol. 2003;77(20):10994-1005. http://dx.doi.org/10.1128/ JVI.77.20.10994-11005.2003

31. Rakoto-Andrianarivelo M, Guillot S, Iber J, Balanant J, Blondel $\mathrm{B}$, Riquet $\mathrm{F}$, et al. Co-circulation and evolution of polioviruses and species $\mathrm{C}$ enteroviruses in a district of Madagascar. PLoS 
Pathog. 2007;3(12):e191. http://dx.doi.org/10.1371/journal. ppat.0030191

32. Ehrenfeld E, Brown D, Jia XY, Summers DF. Antibodies against viral nonstructural proteins in response to infection with poliovirus. J Infect Dis. 1995;171(4):845-50. http://dx.doi. org/10.1093/infdis/171.4.845

33. Cedillo-Barrón L, Foster-Cuevas M, Belsham GJ, Lefèvre F, Parkhouse RM. Induction of a protective response in swine vaccinated with DNA encoding foot-and-mouth disease virus empty capsid proteins and the 3D RNA polymerase. J Gen Virol. 2001;82(Pt 7):1713-24.

34. García-Briones MM, Blanco E, Chiva C, Andreu D, Ley V, Sobrino F. Immunogenicity and T cell recognition in swine of foot-and-mouth disease virus polymerase 3D. Virology. 2004:322(2):264-75. http://dx.doi.org/10.1016/j. virol.2004.01.027

35. Jia XY, Summers DF, Ehrenfeld E. Host antibody response to viral structural and nonstructural proteins after hepatitis $A$ virus infection. J Infect Dis. 1992;165(2):273-80. http://dx.doi. org/10.1093/infdis/165.2.273

36. Jin Y-H, Kang B, Kim BS. Theiler's Virus Infection Induces a Predominant Pathogenic $\mathrm{CD}_{4}+\mathrm{T}$ Cell Response to RNA Polymerase in Susceptible SJL/J Mice. J Virol.

2009;83(21):10981-92. http://dx.doi.org/10.1128/JVI.01398-09

37. Kerkvliet J, Edukulla R, Rodriguez M. Novel roles of the picornaviral 3D polymerase in viral pathogenesis. AdvVirol. 2010;2010:368068.

38. Patel MK, Konde MK, Didi-Ngossaki BH, Ndinga E, Yogolelo R, Salla M, et al. An outbreak of wild poliovirus in the Republic of Congo, 2010-2011.Clin Infect Dis. 2012;55(10):1291-8. http:// dx.doi.org/10.1093/cid/cis714

39. Chumakov K, Ehrenfeld E, Wimmer E, Agol VI. Vaccination against polio should not be stopped. Nature Rev Microbiol. 2007;5(12):952-8. http://dx.doi.org/10.1038/nrmicro1769

40. Global Polio Eradication Initiative. Polio eradication and endgame strategic plan 2013-2018. Geneva: World Health Organization; 2013. p 51-64. Available from: http://www. polioeradication.org/Portals/o/Document/Resources/ StrategyWork/PEESP_EN_US.pdf

41. Anis E, Kopel E, Singer S, Kaliner E, Moerman L, Moran-Gilad J, et al. Insidious reintroduction of wild poliovirus into Israel, 2013. Euro Surveill. 2013;18(38). pii: 20586 